\title{
An unusual manifestation of acute appendicitis with left flank pain
}

\author{
Roland Talanow, MD, PhD \\ The Cleveland Clinic, Department of Radiology, 9500 Euclid Ave., 44195 Cleveland Ohio ( $\triangle-4$ roland@ talanow.net) \\ Radiology Case. 2008 Jul; 2(1):8-11 :: DOI: 10.3941/jrcr.v2i1.27
}

\begin{abstract}
The author presents a case with an unusual presentation of early appendicitis. The patient presented initially with left sided flank pain. Workup for nephrolithiasis, including non-contrast CT of the abdomen and pelvis was negative for renal stones or hydronephrosis. After discharge, the patient presented one week later in the ED with right lower quadrant pain. Contrast enhanced $\mathrm{CT}$ of the abdomen revealed perforated appendicitis.
\end{abstract}

\section{CASE REPORT}

A 42 year old female presented in the ED with left sided pelvic and flank pain that woke her up in the night abruptly. She denied similar symptoms in the past. The patient stated that the pain is better at exam and is worse with activity and certain movements. She denied associated fever, chills, headaches, chest pain, abdominal pain, nausea, vomiting, diarrhea, dysuria or vaginal discharge. The patient is sexually active and denied the possibility of pregnancy. She denied a history of pelvic surgery, ovarian cysts or recent illness. She has not taken anything for the pain. The patient demonstrated on physical examination minimal left adnexal tenderness. There was no discharge or mass, cervix excoriated and no cervical motion tenderness or discharge and no CVA tenderness. Urinalysis and a urine culture were obtained. Urinalysis was positive for: Cloudy/yellow appearance with moderate leukocytes (range: low: 5eg; high: large) and large blood (range: low: neg; high: $3+$ ). Concern for urinary tract infection (UTI) as well as nephrolithiasis was raised and a noncontrast $\mathrm{CT}$ of the abdomen was performed to rule out renal/ureteral stones and urinary obstruction. The exam was negative for stones or hydronephrosis (Fig. 1). Incidental note was made of an appendicolith in a nondilated $(6 \mathrm{~mm})$ appendix or an appendiceal stump (Fig. 2). There was no evidence of diverticulitis or other inflammatory abdominal or pelvic changes. A $2.3 \mathrm{~cm}$ right ovarian cyst and a $2.0 \mathrm{~cm}$ left ovarian cyst were identified as well as a $1.3 \mathrm{~cm}$ coarse calcification anterior to the lower uterine segment, likely a calcified uterine fibroid. Since the urinalysis was suspicious for a UTI, Gynecology was subsequently consulted. The patient was treated for a UTI and received Bactrim DS BID x 3 days (Trimethoprim and Sulfamethoxazole) as well as a prescription for Naproxen $500 \mathrm{mg}$ and was discharged. The final urine culture however did not show any growth.
7 days after initial presentation, the patient presented again in the ED. This time the pain was crampy and in the right lower quadrant, accompanied with nausea. A contrast enhanced CT of the abdomen was performed which demonstrated this time dilatation and inflammation of the appendix with surrounding soft tissue stranding (Fig. 3 and Fig. 4). The previously seen $1 \mathrm{~cm}$ fecolith is again identified in the proximal appendix. Gas bubbles are seen within and outside the appendiceal lumen, medial to the cecum, consistent with perforated appendicitis (Fig. 5). There is associated focal wall thickening of the neighboring distal sigmoid (Fig. 6). Laparoscopy was performed and a gangrenous perforated appendix was found that had an auto-amputated, appendicolith and phlegmon contained in the right lower quadrant. Laparoscopic appendectomy and placement of an intraabdominal drain were performed. Pathology revealed roughened and hemorrhagic serosal surface with an empty lumen. Perforation was identified at the distal portion of the specimen. The postoperative course was unremarkable and the drain in the right pericolic space could be removed on post op day 11 .

\section{DISCUSSION}

The question arises if the initial left flank pain was a "Red Herring" and was not related to the following appendicitis or if this might represent an unusual manifestation of an early appendicitis. Except for the left flank pain, the patient did not have any other associated symptoms, such as nausea or peritonitic signs.

The typical history of appendicitis includes pain starting centrally (periumbilical) before localising to the right iliac fossa; this is due to the poor localizing (spatial) property of visceral nerves from the mid-gut, followed by the involvement of somatic nerves (parietal peritoneum) as the inflammation progresses. The pain is usually associated with loss of appetite and fever. Nausea or 
vomiting may or may not occur. Rovsing's sign is a sign of appendicitis and is elicited by palpation of the lower left quadrant resulting in more pain in the right lower quadrant. However in this patient the pain was located in the left flank and pelvis and no pain was noted in the right abdomen at all.

Catalano et Al. assessed the accuracy of non-contrastenhanced helical CT in offering an alternative explanation for flank pain (Ref. 1). A wide spectrum of findings could be identified in subjects imaged for flank pain. 151 additional CT findings in 77 patients were shown with a $74 \%$ sensitivity and a $99 \%$ specificity for diagnosing alternative causes. 1 case of acute appendicitis within a studied population of 181 patients was detected, however the side of flank pain in that case could not be determined.

On the initial exam, a $1 \mathrm{~cm}$ appendicolith was "incidentally" noted. Appendicoliths may be detected with CT in an otherwise normal appendix (Ref. 2). Appendicoliths are identified by CT in $20-40 \%$ of patients diagnosed with appendicitis (Ref. 3). However, there is limited literature evaluating the predictive values of a diagnosis of appendicitis when CT demonstrated an appendicolith. Another study, which investigated children with suspected appendicitis, showed that an appendicolith detected on CT had a sensitivity of $65 \%$ and a specificity of $86 \%$ for the radiologist diagnosing appendicitis. An appendicolith had a positive predictive value of $74 \%$ and a negative predictive value of $26 \%$ (Ref. 4). The authors of that study concluded that although an appendicolith is significantly associated with appendicitis, the detection of an isolated appendicolith on CT is not sufficiently specific to be the sole basis for the diagnosis of acute appendicitis. Another study followed retrospectively 74 patients with appendicoliths and who did not initially underwent appendectomy. 52 of 74 (70\%) patients had no appendiceal symptoms, were given an alternate diagnosis, and did not return with appendicitis (Ref. 5). 22 of 74 (30\%) patients were discharged without acute appendicitis but with possible appendiceal symptoms. 5 of these $22(23 \%)$ patients returned with pathologically proven acute appendicitis, and all had possible appendiceal symptoms at initial presentation. The authors of that study conclude that an appendicolith may be a marker of increased risk for appendicitis but is not an indication for appendectomy.

\section{TEACHING POINT}

An appendicolith in the setting of left flank pain and no evidence of urinary stones or obstruction may be a sign of early appendicitis.

\section{ABBREVIATIONS}

\author{
$\mathrm{ED}=$ Emergency department \\ $\mathrm{CT}=$ Computed tomography \\ $\mathrm{UTI}=$ Urinary tract infection \\ $\mathrm{CVA}=$ Costovertebral angle \\ $\mathrm{BID}=\mathrm{Bis}$ in die (latin: twice a day)
}

\section{REFERENCES}

1. Catalano O, Nunziata A, Sandomenico F, Siani A . Acute flank pain: comparison of unenhanced helical CT and ultrasonography in detecting causes other than ureterolithiasis. Emerg Radiol. 2002 Sep;9(3):146-54

2. Benjaminov O, Atri M, Hamilton $\mathrm{P}$, Rappaport D . Frequency of visualization and thickness of normal appendix at nonenhanced helical CT. Radiology. 2002 225:400-406

3. Rao PM, Rhea JT, Novelline R . Helical CT incidence and characterization of appendicoliths in 100 patients with appendicitis. Emerg Radiol. 1997 4:55

4. Lowe LH, Penney MW, Scheker LE, Perez R Jr, Stein SM, Heller RM, Shyr Y, Hernanz-Schulman M . Appendicolith Revealed on CT in Children with Suspected Appendicitis. How Specific Is It in the Diagnosis of Appendicitis? AJR Am J Roentgenol. 2000 Oct;175(4):981-4

5. Rabinowitz CB, Egglin TK, Beland MD, Mayo-Smith WW . Outcomes in 74 patients with an appendicolith who did not undergo surgery: is follow-up imaging necessary? Emerg Radiol. 2007 Jul;14(3):161-5. Epub 2007 Apr 25

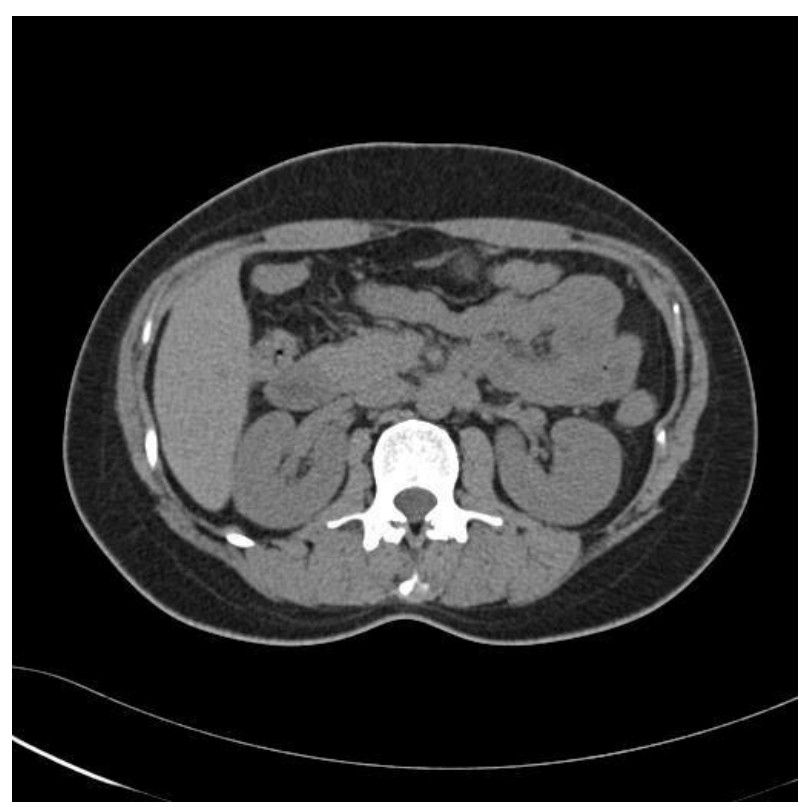

Figure 1: Computed Tomography

Noncontrast CT of the abdomen was negative for stones or hydronephrosis. 


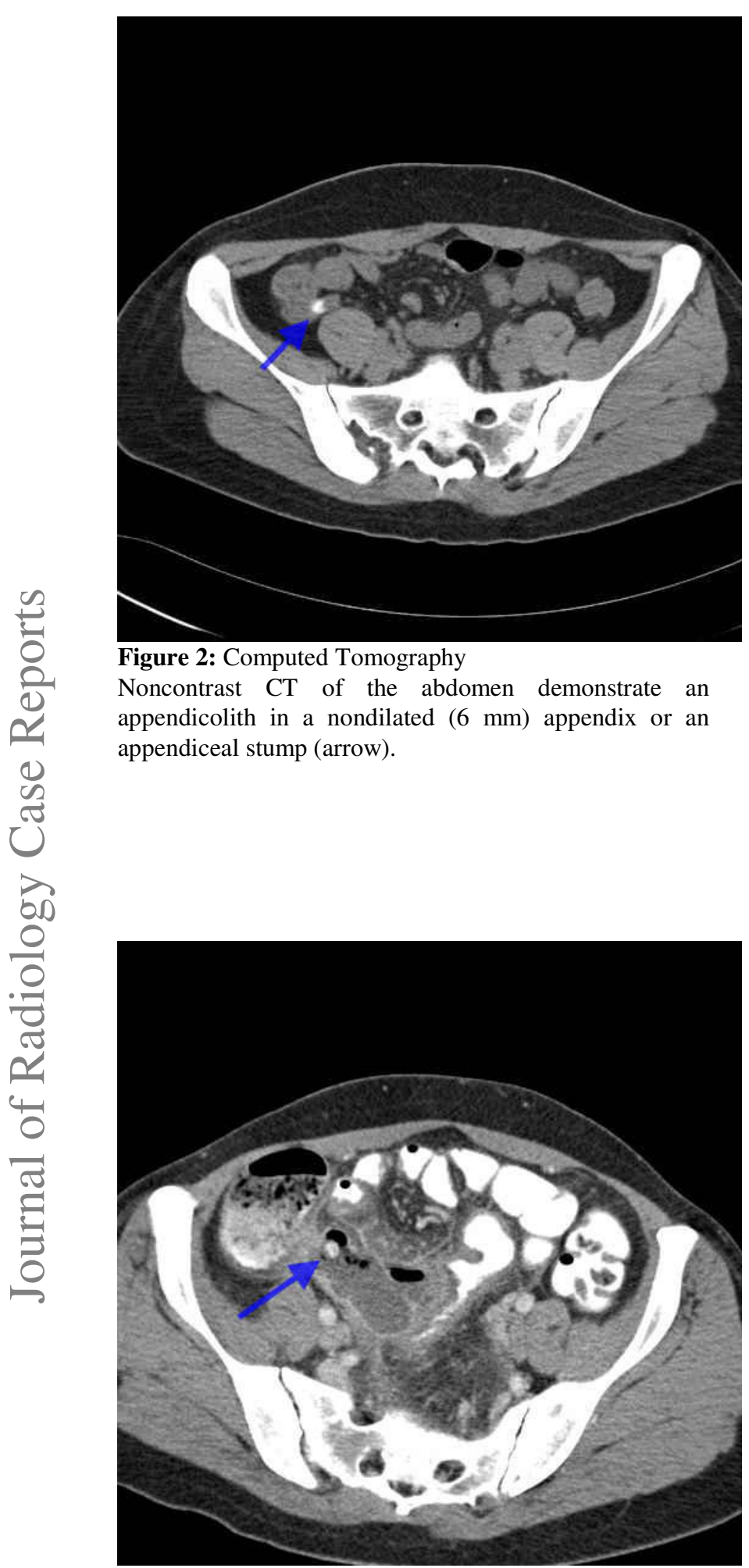

Figure 3: Computed Tomography

Contrast enhanced axial CT of the abdomen demonstrates dilatation and inflammation of the appendix with surrounding soft tissue stranding. Gas bubbles are seen within and outside the appendiceal lumen, medial to the cecum, consistent with perforated appendicitis. The previously seen $1 \mathrm{~cm}$ fecolith is again identified in the proximal appendix (arrow).

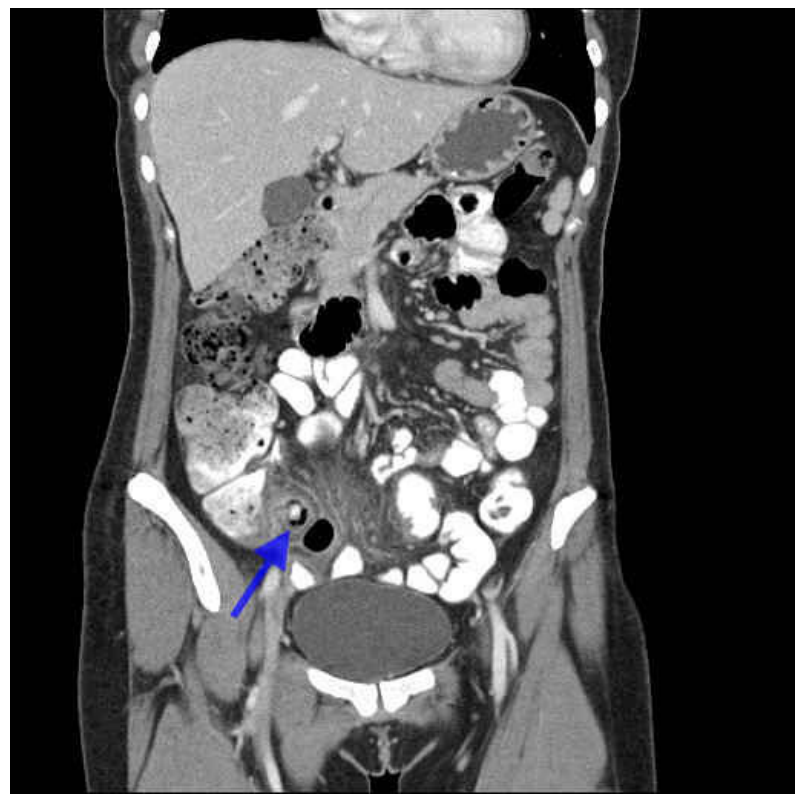

Figure 4: Computed Tomography

Contrast enhanced coronal reformatted CT of the abdomen demonstrates dilatation and inflammation of the appendix with surrounding soft tissue stranding and gas bubbles within and outside the appendiceal lumen, medial to the cecum, consistent with perforated appendicitis. The previously seen $1 \mathrm{~cm}$ fecolith is again identified in the proximal appendix (arrow).

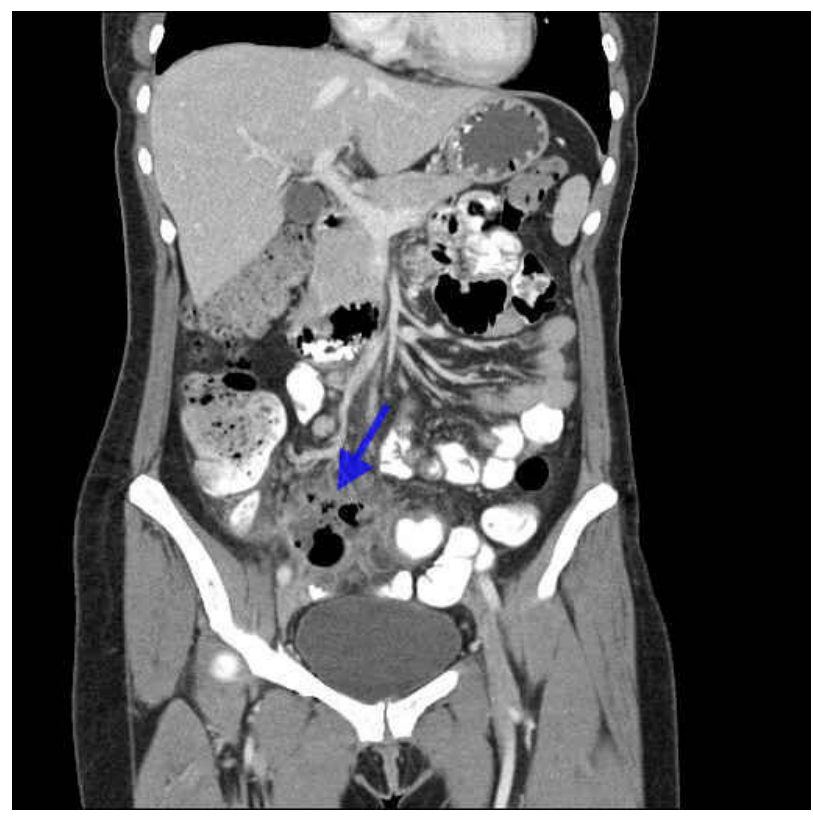

Figure 5: Computed Tomography

Contrast enhanced coronal reformatted $\mathrm{CT}$ of the abdomen demonstrates gas bubbles (arrow) within and outside the appendiceal lumen, medial to the cecum, consistent with perforated appendicitis. 


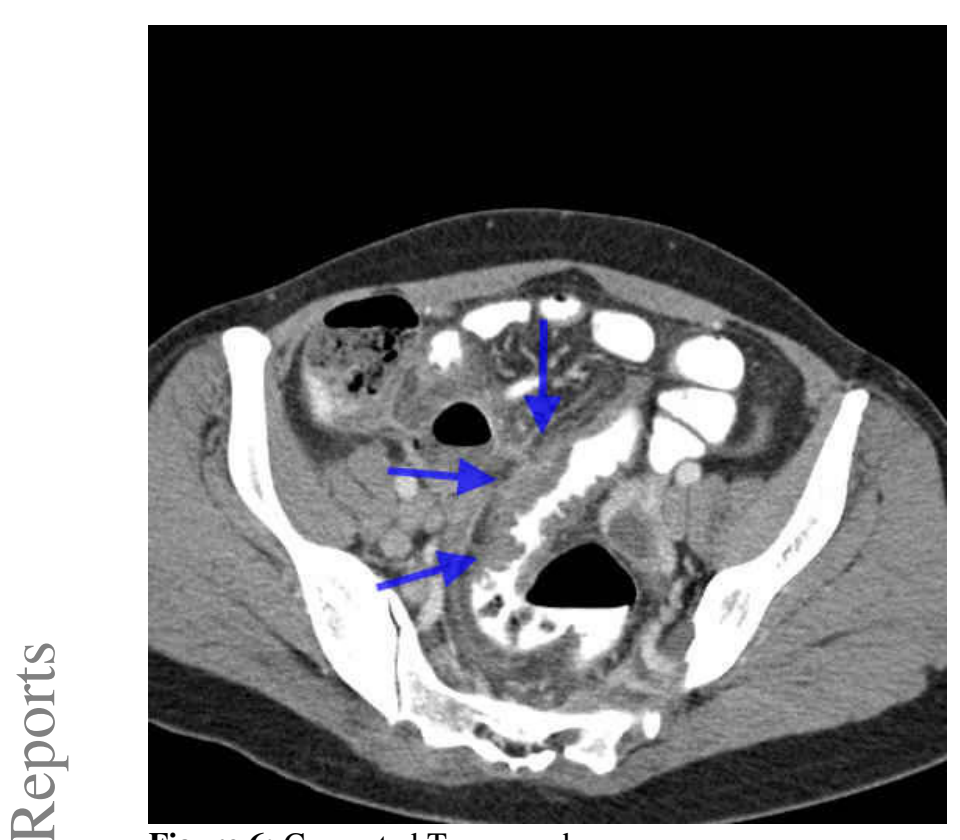

Figure 6: Computed Tomography

(1) Contrast enhanced axial CT of the abdomen demonstrates

of circumferential focal wall thickening of the distal sigmoid

$U$ (arrows) which is affected by the inflammatory changes

of the appendix.

URL of this article:

www.radiologycases.com/index.php/radiologycases/article/view/27

Published by EduRad

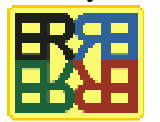

www.EduRad.org 